\title{
Relação entre uso do telefone celular antes de dormir, qualidade do sono e sonolência diurna
}

\author{
Correlation between mobile phone use before sleep, \\ sleep quality and daytime sleepiness
}

\section{Carine Cristina Moraes de Freitas ${ }^{1}$, Agda Lopes Donnabella Marconi Gozzoli ${ }^{1}$, Juliana Naomi Konno', Vera Lucia Ribeiro Fuess²}

Freitas CCM, Gozzoli ALDM, Konno JN, Fues VLR. Relação entre uso do telefone celular antes de dormir, qualidade do sono e sonolência diurna / Correlation between mobile phone use before sleep, sleep quality and daytime sleepiness. Rev Med (São Paulo). 2017 jan.-mar;;96(1):14-20.

RESUMO: OBJETIVO: Verificar alteração na qualidade e duração do sono, assim como na sonolência diurna após os alunos se absterem do uso do telefone celular próximo ao horário de dormir. METODOLOGIA: Comparou-se a mesma população de estudantes de medicina em duas ocasiões: em uso normal do aparelho e após ficar sem usá-lo uma hora antes de dormir durante 15 dias. A amostra foi composta por 76 estudantes de medicina, do $1^{\circ}$ ao $4^{\circ}$ ano, com idades entre 17 e 40 , que se voluntariaram a participar da pesquisa e assinaram o TCLE. Como instrumentos de coleta de dados, foram utilizados os questionários: Pittsburgh Sleep Quality Index (PSQI), Escala de Sonolência de Epworth (ESE) e um instrumento para avaliar o uso do celular desenvolvido para esta pesquisa. Para análise estatística, foi utilizado o teste t-student pareado não-paramétrico, sendo adotado um $\mathrm{p}<0,05$ como nível de significância. RESULTADOS: Foram observadas mudanças significativas nos resultados do PSQI $(p<0,01)$ com melhora da qualidade do sono em $65,7 \%$ dos participantes (média pré $8,2 \pm 2,9$ /média pós $6,4 \pm 2,7)$. Também se observou mudança significativa nos escores da ESE $(p<0,01)$, com diminuição da sonolência diurna em $85,5 \%$ dos participantes (média pré $8.9 \pm$ 3,3/média pós $5,1 \pm 2,7$ pontos). DISCUSSÃO: Houve significativa melhora tanto na qualidade do sono dos voluntários quanto na sonolência diurna. Um importante fator a ser considerado é que a restrição dada aos voluntários foi somente com relação ao uso do celular, não estando incluídos outros aparelhos eletrônicos. Também não foi levada em consideração neste estudo a ansiedade gerada pela intervenção. Ambos os fatores podem ter relação com os casos de piora ou manutenção dos escores. Com a restrição do uso do aparelho celular por uma hora antes de dormir por um período de 15 dias, foi possível notar mudanças estatisticamente significativas no sono dos participantes.

Descritores: Telefones celulares; Sono; Distúrbios do início e da manutenção do sono; Distúrbios do sono por sonolência excessiva.

ABSTRACT: OBJECTIVE Verify changes on sleep quality and duration after abstention from the use of mobile phones close to sleep time, such as decrease of daytime sleepiness. METHODOLOGY: The same population of medical students was compared on two occasions: during normal use of the device

Instituição em que foi realizado o trabalho: Universidade de Mogi das Cruzes, Faculdade de Medicina, Mogi das Cruzes, SP. Apresentado no Congresso da Associação Brasileira do Sono, São Paulo, SP, 12 dez. 2016.

1. Acadêmicas do quarto ano da Faculdade de Medicina da Universidade de Mogi das Cruzes. E-mail: cafreitas2@gmail.com, agda_ donnabella@hotmail.com, juliananaomi224@gmail.com.

2. Orientadora; Professora adjunta da Universidade de Mogi das Cruzes; Doutora em Otorrinolaringologia pela Universidade de São Paulo; com certificação em Medicina do Sono pela Associação Brasileira de Medicina do Sono. E-mail: verafuess@terra.com.br. Endereço para correspondência: Carine Cristina Moraes de Freitas. Endereço: Rua Ernesto Evans, 578, ap. 76, São Miguel Paulista, CEP 08021-170 São Paulo, SP. 
and after not using it an hour before bedtime for 15 days. The sample consisted of 76 medical students from 1st to 4th year, aged 17 to 40 years, who volunteered to participate in the study and signed a consent form. As data collection instruments, the following questionnaires were used: Pittsburgh Sleep Quality Index (PSQI), Epworth Sleepiness Scale (ESS) and a tool to assess the use of mobile phones developed for this research. The statistical analysis was performed using the t-student's test paired nonparametric, adopting $\mathrm{p}<0.05$ as significance level. RESULTS: Significant changes were observed on PSQI results $(\mathrm{p}<0.01)$ with a sleep quality increase in $65.7 \%$ of the participants (mean score before $8.2 \pm 2.9 /$ mean score after $6.4 \pm 2.7$ ). There were also significant changes on the Epworth Sleepiness Scale $(\mathrm{p}<0.01)$, with a decrease of daytime sleepiness in $85.5 \%$ of the

\section{INTRODUÇÃO} sono tem um papel fundamental na saúde e
no bem-estar das pessoas. A qualidade do sono tem influência sobre a saúde mental, a saúde física e a qualidade de vida do indivíduo. Seu papel nas funções biológicas inclui desde a consolidação da memória ${ }^{1}$ até a restauração do metabolismo energético cerebral ${ }^{2}$.

\section{Regulação do sono}

A regulação do sono é feita a partir de três principais fatores. O primeiro fator, o homeostático, representa a "necessidade de dormir", estando provavelmente vinculada ao metabolismo da adenosina e sua interação com os neurônios colinérgicos ${ }^{3}$. O segundo, o fator circadiano, influencia principalmente o horário em que se dorme, relacionando-se à sincronização com o ciclo claro-escuro do ambiente 4 . Por último, o fator comportamental, que se sobrepõe aos fatores homeostático e circadiano. Sua influência é notada principalmente em hábitos próximos à hora de dormir, podendo acarretar sérios distúrbios do sono ${ }^{5}$.

A privação prolongada do sono, ou seja, um longo período sem dormir ou de sono fragmentado, pode acarretar em qualidade ruim de sono $^{6}$ e em sonolência excessiva durante o período de vigília ${ }^{7}$.

\section{Qualidade do sono e uso do celular}

Nas últimas décadas, principalmente com o advento da acessibilidade e portabilidade das diversas mídias, a sociedade tem sido protagonista de um largo aumento no uso de aparelhos eletrônicos. À medida que esses aparelhos se tornam mais leves e portáteis, tem sido crescente o número de pessoas que os levam a qualquer lugar, inclusive para cama ${ }^{8}$.

Um estudo do IBOPE Media revela que 134 milhões de pessoas (67,4\% da população), com idade maior que 10 anos, têm telefone móvel no Brasil. Dentre eles, 52 milhões têm acesso à internet pelo celular (smartphone), o que participants (mean before $8.9 \pm 3.3 /$ mean score after $5.1 \pm 2.7$ points). DISCUSSION: There was significant improvement in sleep quality and daytime sleepiness. An important factor to be considered is that the restriction applied to the volunteers was only related to mobile phone use, other electronic devices were not included. The anxiety caused by the intervention was also not considered. Both factors might be related to the cases in which there was a worsening or maintenance of the scores. With the restriction of mobile phone on the hour preceding sleep, for 15 days, positive changes on sleep could already be observed.

Keywords: Cell phones; Sleep; Sleep initiation and maintenance disorders; Disorders of excessive somnolence.

representa $38,8 \%$ da população que possui um aparelho celular. $\mathrm{O}$ aparelho celular é mais frequentemente usado para conversar com amigos (76\%), ler e enviar e-mails (75\%), interar-se sobre as notícias $(64 \%)$ e sobre o cenário musical $(47 \%)$ e assistir a vídeos $(44 \%)^{9}$.

Estudos anteriores mostraram também que pessoas que leem livros em aparelhos eletrônicos, em comparação com aquelas que o fazem em livros, levam mais tempo para pegar no sono, têm uma regulação mais tardia do ciclo circadiano e diminuição do estado de alerta no dia seguinte, além de terem alteração na secreção hormonal de melatonina ${ }^{10}$.

\section{Interrupções do Sono e Estresse}

$\mathrm{O}$ uso de smartphones à noite interrompe o sono e está associado a um maior desgaste e menor engajamento durante as atividades do dia seguinte ${ }^{11}$. Quanto maior o uso do celular, maior a deterioração da qualidade do sono ${ }^{12}$.

A demanda gerada pelo celular de que a pessoa esteja sempre disponível para entrar em contato e ao alcance das outras pessoas independentemente do tempo e do espaço pode ser considerada um fator de estresse. Essa acessibilidade implica na possibilidade de ser incomodado a qualquer hora, inclusive durante as horas de sono ${ }^{13}$.

\section{Interferência bioquímica}

Autores sugerem que uso de mais de um aparelho eletrônico por longos períodos de tempo pode diminuir a produção de melatonina, responsável pela regulação hormonal do $\operatorname{sono}^{14}$. A melatonina, um hormônio da glândula pineal que participa do controle do ciclo circadiano, é liberada durante o anoitecer e induz o sono. A exposição à luz, no entanto, inibe sua produção ${ }^{15}$. A luz, mesmo que bem menos intensa que emitida pela iluminação interna da casa, pode suprimir a produção de melatonina ${ }^{16}$.

\section{Presente estudo}

Sabendo que a exposição prolongada à luz de telas 
de aparelhos celulares altera a produção e secreção dos hormônios relacionados aos estados de vigília e de sono, nota-se a importância contemporânea de se investigar os efeitos negativos que tais aparelhos têm sobre a duração e qualidade do sono.

Neste estudo, a população foi de acadêmicos de medicina, que possuem naturalmente sua qualidade do sono alterada devido às elevadas cargas horárias de estudo e trabalho. Dessa forma, é interessante buscar hábitos que melhorem seu sono e aumentem sua disposição diária, como a diminuição do uso do celular antes de dormir.

Para tal fim, realizou-se este estudo do tipo experimental não controlado com delineamento antes e depois, que buscou, através de uma análise comparativa, verificar se houve melhora na qualidade do sono e na sonolência diurna após o período de 15 dias sem fazer uso do telefone celular por no mínimo uma hora antes de ir dormir.

\section{MATERIAL E MÉTODOS}

\section{Participantes}

A amostra foi composta por um número $\mathrm{n}$ de 76 estudantes de medicina, do $1^{\circ}$ ao $4^{\circ}$ ano, com idades entre 17 e 40 anos da Universidade de Mogi das Cruzes, que se voluntariaram a participar da pesquisa e assinaram o TCLE.

\section{Instrumentos}

Como instrumentos de coleta de dados, foram utilizados os seguintes questionários: Pittsburgh Sleep Quality Index (PSQI), Escala de Sonolência de Epworth (ESE) e um instrumento para avaliar o uso do celular desenvolvido para esta pesquisa. Foi utilizado um aplicativo de mensagens instantâneas para celulares, WhatsApp ${ }^{\circledR}$, no qual foi criado um grupo incluindo todos os participantes da pesquisa.

\section{Índice de qualidade do sono de Pittsburgh (PSQI)}

Nesse estudo foi utilizada a versão validada em português brasileiro do Índice de qualidade do sono de Pittsburgh, a qual é um instrumento confiável e equivalente à sua versão original ${ }^{15}$. O Índice de qualidade do sono de Pittsburgh é um instrumento utilizado para medir a qualidade do sono através da análise de 7 componentes: qualidade do sono, latência do sono, duração do sono, eficiência habitual do sono, alterações do sono, uso de medicações para o sono e disfunção diurna. Esses sete componentes são avaliados através de 19 itens que incluem perguntas de múltipla escolha, assim como itens que requerem respostas qualitativas dos participantes. Esse instrumento gera uma pontuação que varia entre $0-21$; uma pontuação maior que 5 indica qualidade ruim do sono ${ }^{16}$.

\section{Escala de Sonolência de Epworth (ESE)}

Foi utilizada a versão validada em Português para uso no Brasil da Escala de Sonolência de Epworth ${ }^{17}$. Tratase de um questionário autoaplicável que avalia a sonolência diurna através da análise da probabilidade de adormecer em oito situações envolvendo atividades diárias comuns ${ }^{18}$. O participante atribui notas de 0 a 3 para cada uma das 8 situações sendo que uma nota 0 indica que ele nunca cochilaria e uma nota 3 indica que há grande probabilidade de cochilar naquela situação. Essas pontuações são somadas e compõem uma pontuação global que varia entre 0-24. Uma pontuação maior que 10 sugere diagnóstico de sonolência diurna excessiva ${ }^{18,19}$. celular

Instrumento para avaliar os hábitos de uso do

Esse instrumento foi criado pelos pesquisadores, com o objetivo de avaliar qualitativamente os hábitos de uso do celular imediatamente antes de dormir e durante o sono através de perguntas auto-avaliativas com respostas sim ou não sobre os hábitos de uso do celular dos participantes.

\section{Grupo no aplicativo Whatsapp ${ }^{\circledR}$}

Foi criado um grupo no aplicativo para celulares Whatsapp ${ }^{\circledR}$, para enviar mensagens diárias aos participantes, solicitando que desligassem os celulares. Esse grupo também era utilizado para tirar dúvidas dos participantes e para confirmação de que os indivíduos estavam desligando o celular. Caso o participante relatasse falha ao desligar o celular, era solicitado que reiniciasse a intervenção, caso contrário, seus resultados eram descartados.

\section{Procedimento}

Os voluntários receberam todas as informações necessárias sobre o estudo e assinaram o Termo de Consentimento Livre e Esclarecido (TCLE). Tanto o termo de consentimento como os instrumentos utilizados neste estudo foram aprovados pelo Comitê de Ética da Universidade de Mogi das Cruzes, sob o número CAAE 37570114.2.0000.5497. Os participantes foram avaliados através dos instrumentos citados em dois momentos distintos, antes da intervenção e depois. Na primeira ocasião, os participantes responderam aos três questionários citados anteriormente, de modo a avaliar a qualidade do sono, a sonolência diurna e seus hábitos de uso do celular. Após essa primeira avaliação, foi iniciada a intervenção, na qual foi pedido aos voluntários que ficassem sem utilizar o celular durante uma hora antes de dormir todos os dias, durante 15 dias. Terminados esses 15 dias, os participantes foram avaliados novamente através do PSQI e da Escala de Sonolência de Epworth, e os resultados foram comparados com aqueles da primeira avaliação para verificar se houve melhora na qualidade do sono e na sonolência diurna. 


\section{Análise de dados}

A análise dos dados foi feita de acordo com os escores dos testes pré-determinados, sendo a comparação feita através do teste t-student pareado não-paramétrico. Foi adotado um $\mathrm{p}<0,05$ como nível de significância.

\section{Limitações do estudo}

A população definida para este estudo não apresentou um grupo controle composto por alunos que não sofreram a intervenção. $\mathrm{O}$ estudo não foi randomizado, sendo feito medidas somente pré e pós intervenção.

Os participantes foram informados sobre o intuito da pesquisa, de modo que os indivíduos talvez tivessem a tendência a tentar apresentar resultados melhores por saberem qual é a ideia do estudo, embora se trate de uma população que tem consciência da importância da imparcialidade em pesquisas.

\section{RESULTADOS}

\section{Perfil sociodemográfico da amostra dos 76 estudantes}

Tabela 1. Perfil sociodemográfico dos estudantes de medicina $(\mathrm{n}=76)$

\begin{tabular}{lcc}
\hline Variável & $\mathbf{N}$ & $\mathbf{\%}$ \\
\hline Gênero & & \\
$\quad$ Masculino & 18 & 23,7 \\
$\quad$ Feminino & 58 & 76,3 \\
Idade & & \\
$\quad$ 17-24 anos & 58 & 76,3 \\
25 ou mais & 15 & 19,8 \\
$\quad$ Ignorado & 3 & 3,9 \\
Ano de graduação & & \\
$1^{\mathbf{0}}$ & 20 & 26,3 \\
$2^{\mathbf{0}}$ & 15 & 19,7 \\
$3^{\mathbf{0}}$ & 17 & 22,4 \\
$4^{\mathbf{0}}$ & 24 & 31,6 \\
\hline
\end{tabular}

As análises dos dados mostram que 70 (93\%) alunos voluntários mantêm o celular próximo, e que 57 (76\%) o utilizam mesmo após se deitarem. Observou-se que 51 (68\%) participantes acordam caso o celular toque. Além disso, $60(79 \%)$ participantes utiliza o celular por pelo menos 15 minutos após se deitar.

A partir dos dados coletados, observou-se que a maioria dos voluntários continua a utilizar o celular após se deitar e o mantém próximo da cama ao dormir. É importante a correlação desses dados com a qualidade do sono e a sonolência diurna.

\section{Duração do sono}

A duração do sono foi um dos critérios avaliados para determinar o escore final do Índice de Qualidade do Sono de Pittsburgh (PSQI). Ao avaliar os resultados obtidos antes e depois dos voluntários se absterem do uso do celular próximo ao horário de dormir, observou-se que a média da duração do sono aumentou de $6,6 \pm 1,1 \mathrm{~h}$, para $7,2 \pm 0,8 \mathrm{~h}$ (Figura 1), após os voluntários se absterem do uso do celular próximo ao horário de dormir por 15 dias, demonstrando um melhora pontual em um dos quesitos analisados pelo questionário $(\mathrm{p}<0,01)$.

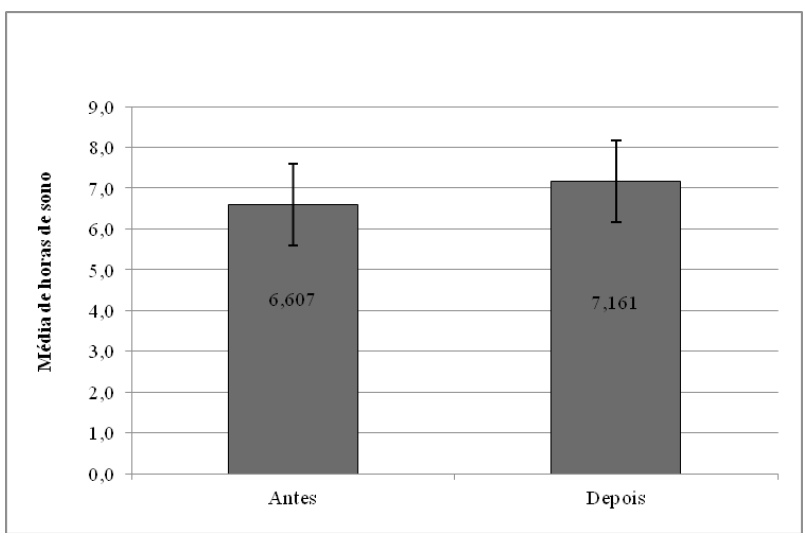

Figura 1. Média da duração do sono em horas, com desvio padrão, pré e pós-abstenção do celular por 15 dias

\section{Impacto da intervenção sobre a sonolência diurna}

A Escala de Sonolência de Epworth (ESE) classifica a probabilidade de cochilar, tendo a pontuação maior que 10 como ponto de corte, maior que 16 indicando sonolência grave e pontuações baixas indicando pouca propensão para dormir.

Observou-se na primeira fase 27 (35\%) voluntários com escore acima do ponto de corte e na segunda fase apenas $4(5 \%)$ voluntários permaneceram com a pontuação acima de 10 pontos, demonstrado na Figura 2. Comparando a pontuação final da ESE entre as duas fases, verificou-se uma redução dos escores em 65 (86,7\%) voluntários.

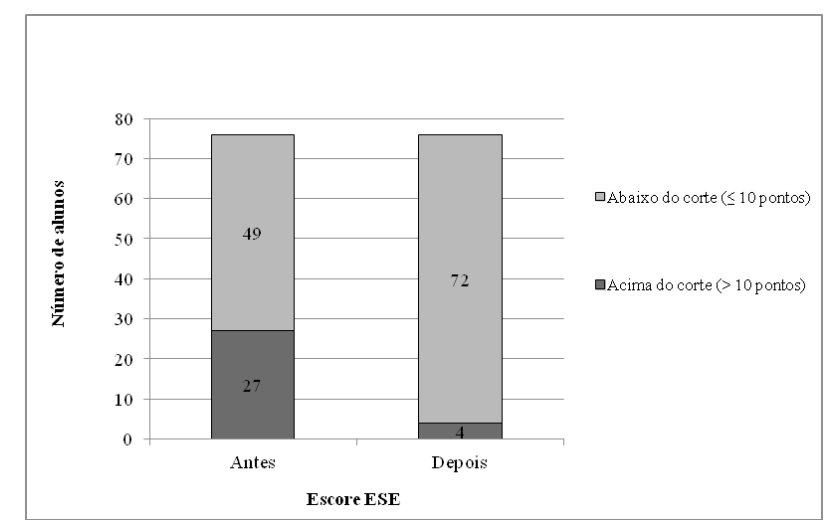

Figura 2. Número de alunos que obtiveram pontuação acima ou abaixo de 10 pontos na ESE, antes e depois da intervenção 
Ao analisar os resultados obtidos com a aplicação da ESE antes (primeira fase) e após a abstinência do celular antes de dormir (segunda fase), a média das pontuações na primeira fase foi de $8,9 \pm 3,3$ pontos, e na segunda fase foi de $5,1 \pm 2,7$ pontos $(\mathrm{p}<0,01)$.
A Figura 3 apresenta a distribuição do número de alunos por escore. Verifica-se que a distribuição obedece a uma curva gaussiana, tanto antes quanto após a abstinência do celular antes de dormir. Percebe-se que houve deslocamento da curva para a esquerda após a intervenção.

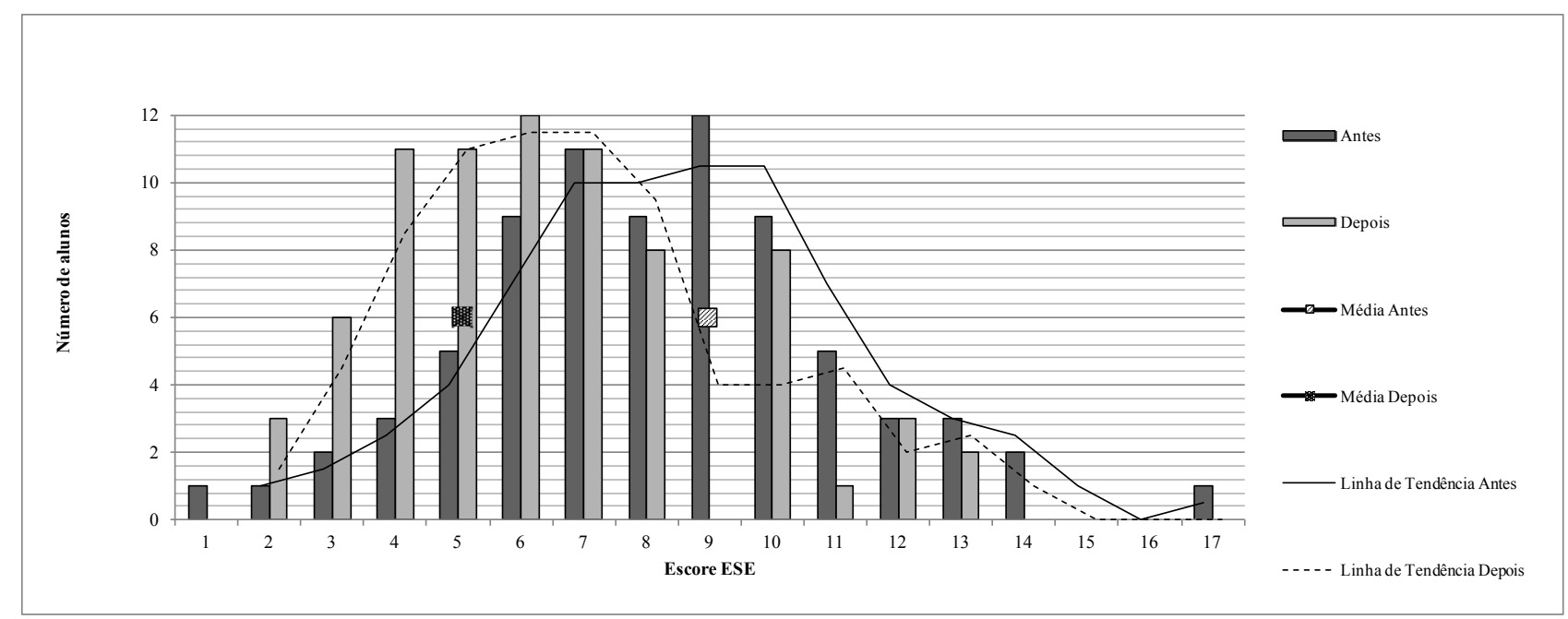

Figura 3. Curvas de distribuição do escore ESE em função do número de alunos por escore. Barras cinzas: antes da abstinência do celular antes de dormir; barras brancas: depois da abstinência do celular antes de dormir. Barras de outras cores: médias

Impacto da intervenção sobre a qualidade do sono

No Índice de Qualidade do Sono de Pittsburgh (PSQI) os componentes somados geram uma pontuação que varia entre 0 (zero) e 21 pontos, quanto menor a pontuação melhor a qualidade do sono e pontuações maiores que 5 sugerem qualidade ruim do sono.

As análises dos dados do PSQI mostraram uma

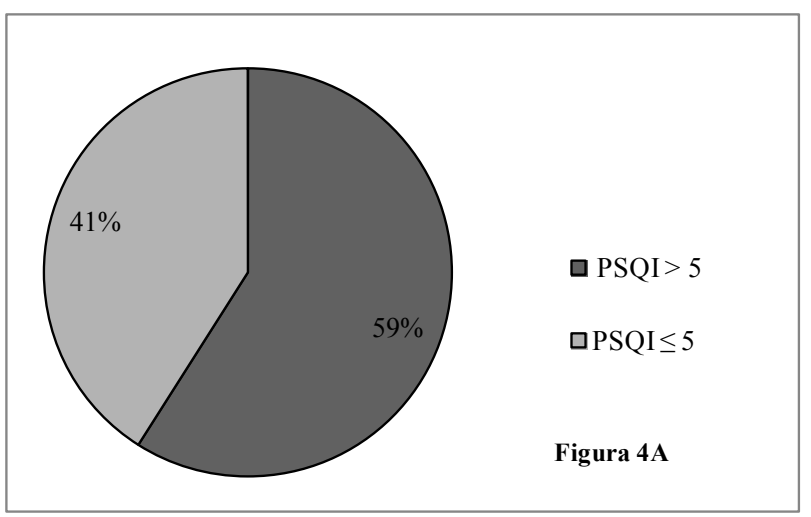

diminuição nos escores entre a primeira e a segunda fase. A média passou de $8,2 \pm 2,9$, para $6,4 \pm 2,7(\mathrm{p}<0,01)$. Na primeira fase, $64(84 \%)$ voluntários apresentavam escore final maior que 5 (Figura 4A), indicando uma qualidade ruim do sono, enquanto que na segunda fase (fase pós abstinência), esse número diminuiu para 45 (59\%) voluntários (Figura 4B).

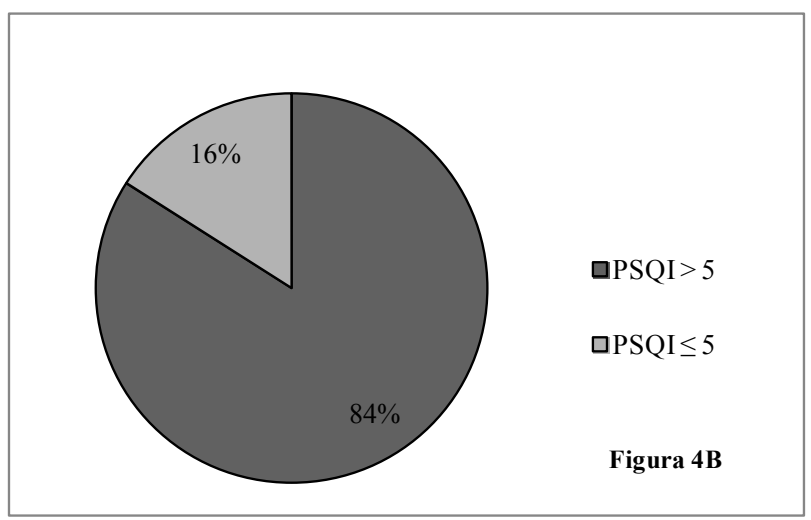

Figura 4. Distribuição dos voluntários em relação à qualidade do sono de acordo com o escore PSQI. A: primeira fase. B: segunda fase

Comparando a pontuação final do PSQI entre as duas fases, verificou-se uma redução dos escores em 50 $(66 \%)$ voluntários.

Vale observar que $9,2 \%$ dos voluntários não apresentaram mudanças nos escores, e em $25 \%$ constatouse piora dos escores do PSQI.

\section{DISCUSSÃO}

Pesquisas anteriores apontam que a abstinência do uso de celulares por uma hora antes de dormir, ocasiona melhora nos parâmetros qualidade do sono e sonolência diurna $^{11}$, uma vez que o uso do celular antes de dormir está 
relacionado a uma pior qualidade do sono na mesma noite. Avaliações comparativas entre acadêmicos e adultos em geral mostram uma pior qualidade de sono e maior sonolência diurna no grupo de acadêmicos, eventos estes diretamente relacionados com queda no rendimento acadêmico $^{7,20}$.

Em função desses apontamentos e da necessidade de otimização da atenção e disposição dos estudantes da área da saúde, avaliou-se se a abstinência de celular por pelo menos uma hora antes de dormir promoveria uma melhora na qualidade do sono e redução da sonolência diurna.

Para tal finalidade, foram aplicados os questionários padronizados PSQI e ESE, os quais avaliam, respectivamente, a qualidade do sono e a sonolência diurna.

A análise comparativa dos resultados obtidos mostrou que houve significativa melhora tanto na qualidade do sono quanto na sonolência diurna dos voluntários, uma vez que verificou-se redução nos escores do PSQI e da ESE dos voluntários em $66 \%$ e $86,6 \%$, respectivamente.

Esses dados do PSQI corroboram que a abstenção do celular antes de dormir promove uma melhora na qualidade do sono, contribuindo para uma menor sonolência diurno no dia seguinte, como sugerido pela análise da ESE.

Um importante fator a ser considerado é que a restrição dada aos voluntários foi somente com relação ao uso do celular, não estando incluídos outros aparelhos eletrônicos, como tablets, computadores, televisores, que também poderiam influenciar a qualidade do sono devido à luz por eles emitida. Também não foi levada em consideração neste estudo a ansiedade gerada pela abstinência do celular mantido desligado por uma hora imediatamente antes de dormir. A manifestação de ansiedade, fator que influencia na qualidade do sono, bem como a substituição do celular por outros aparelhos eletrônicos, podem estar diretamente relacionados aos casos de piora ou não melhora, alterando os escores em alguns casos do estudo.

\section{CONCLUSÃO}

Conclui-se que a restrição do uso do aparelho celular por uma hora antes de dormir pelo período de 15 dias promoveu melhora na qualidade do sono dos participantes, bem como redução da sonolência diurna. Entretanto, mais estudos devem ser conduzidos com o objetivo de avaliar o uso de outros aparelhos eletrônicos geradores de luz antes de dormir, assim como a ansiedade possivelmente gerada pela abstinência do uso desses aparelhos.

Agradecimentos: Agradecemos aos Profs. Drs. Edgar Julian Paredes Gamero e Wagner Alves de Souza Júdice pelas orientações fornecidas no que diz respeito à parte estatística e revisão final do texto deste artigo.

\section{REFERÊNCIAS}

1. Reimão R. Sono: estudo abrangente. 2a ed. São Paulo: Atheneu; 1996.

2. Ferrara M, Gennaro L, How much sleep do we need? Sleep Med Rev. 2001;5(2):155-79. doi: 10.1053/smrv.2000.0138.

3. Bjorness TE, Greene RW. Adenosine and sleep. Curr Neuropharmacol. 2009;7(3):238-45. doi: 10.2174/157015909789152182.

Borbély AA, Achermann P. Sleep homeostasis and models of sleep regulation. J Biol Rhythms. 1999;14(6):557-68.

4. Dijk DJ, Czeisler CA. Contribution of the circadian pacemaker and the sleep homeostat to sleep propensity, sleep structure, electroencephalographic slow waves, and sleep spindle activity in humans. J Neurosci. 1995;15(5):3526-38. Available from: http://www.jneurosci.org/content/jneuro/15/5/3526.full. pdf.

5. Bjorvatn B, Pallesen S. A practical approach to circadian rhythm sleep disorders. Sleep Med Rev. 2009;13:47-60. doi: 10.1016/j.smrv.2008.04.009.

6. Dorrian J, Dinges DF. Sleep deprivation and its effects on cognitive performance. In: Encyclopedia of sleep medicine. New Jersey: John Wiley and Sons; 2005. p.139-44. doi: http:// www.ncbi.nlm.nih.gov/pmc/articles/PMC2656292/.

7. Araújo DF, Almondes KM. Qualidade de sono e sua relação com o rendimento acadêmico em estudantes universitários de turnos distintos. Rev Psico. 2012;43:350-9. Disponível em: $\mathrm{http} / / /$ revistaseletronicas.pucrs.br/ojs/index.php/revistapsico/ article/view/9369/8236.

8. Fossum IN, Nordnes LT, Storemark SS, Bjorvatn B, Pallesen $\mathrm{S}$. The Association between use of electronic media in bed before going to sleep and insomnia symptoms, daytime sleepiness, morningness, and chronotype. Behav Sleep Med. 2014;12(5):343-57. doi: 10.1080/15402002.2013.819468.

9. IBOPE. Milhões de pessoas têm acesso à web pelo celular, aponta IBOPE Media [citado 3 maio 2016]. Disponível em: http://www.ibope.com.br/pt-br/noticias/paginas/52-milhoesde-pessoas-tem-acesso-a-web-pelo-celular-aponta-ibopemedia.aspx.

10. Chang AM, Aeschbacha D, Duffya JF, Czeislera CA. Evening use of light-emitting eReaders negatively affects sleep, circadian timing, and next-morning alertness. Proc Nat Acad Sci. 2015;112:1232-7. doi: 10.1073/pnas.1418490112.

11. Lanaj K, Johnson RE, Barnes MN. Beginning the workday yet already depleted? Consequences of late-night smartphone use and sleep. Org Behav Human Decision Processes. 2014;124:11-23. doi: 10.1016/j.obhdp.2014.01.001.

12. Sahin S, Ozdemir K, Unsal A, Temiz N. Evaluation of mobile phone addiction level and sleep quality in university students. Pakistan J Med Sci. 2013;29:913-8. doi: http://www.ncbi.nlm. nih.gov/pmc/articles/PMC3817775/.

13. Thomée S, Härenstam A, Hagberg M. Mobile phone use and stress, sleep disturbances, and symptoms of depression among young adults--a prospective cohort study. BMC Public Health. 
2011;11:66. doi: 10.1186/1471-2458-11-66.

14. Calamaro JC, Mason TBA, Ratcliffe SJ. Adolescents living the 24/7 lifestyle: effects of caffeine and technology on sleep duration and daytime functioning. Pediatrics. 2009;123:100510. doi: 10.1542 /peds.2008-3641.

15. Bertolazi AN, Fagondes SC, Hoff LS, Dartora EG, Miozzo IC, de Barba ME, Barreto SS. Validation of the Brazilian Portuguese version of the Pittsburgh Sleep Quality Index. Sleep Med. 2011;12:70-5. doi: 10.1016/j.sleep.2010.04.020.

16. Buysse DJ, Reynolds $3^{\text {rd }}$ CF, Monk TH, Berman SR, Kupfer DJ. The Pittsburgh, Sleep Quality Index: a new instrument for psychiatric practice and research. Psychiatry Res. 1989;28:193-213. doi: http://www.ncbi.nlm.nih.gov/ pubmed/2748771.

17. Bertolazi AN, Fagondes SC, Hoff LS, Pedro VD, Menna SBS, Johns MW. Validação da escala de sonolência de

Recebido: 12.10 .2016

Aceito: 20.02.2017
Epworth em português para uso no Brasil. J Bras Pneumol. 2009;35:877-83. doi: http://dx.doi.org/10.1590/S180637132009000900009 .

18. Johns MW. A new method for measuring daytime sleepiness: the Epworth sleepiness scale. Sleep. 1991;14:540-5. doi: http://www.ncbi.nlm.nih.gov/pubmed/1798888.

19. Johns MW. Sensitivity and specificity of the multiple sleep latency test (MSLT), the maintenance of wakefulness test and the Epworth sleepiness scale: failure of the MSLT as a gold standard. J Sleep Res. 2000;9:5-11. doi: http://www.ncbi.nlm. nih.gov/pubmed/10733683.

20. Cardoso HC, Bueno FCC, Mata JC, Alves APR, Jochims I, Filho IHRV, Hanna MM. Avaliação da qualidade do sono em estudantes de Medicina. Rev Bras Educ Med. 2009;33:349-55. doi: http://dx.doi.org/10.1590/S0100-55022009000300005. 